\title{
Many-Body-Gravitational Solutions for Galactic Rotational Velocities, Including Possible Negative Mass
}

\author{
Antony J. Bourdillon \\ UHRL, San Jose, CA, USA \\ Email: bourdillona@sbcglobal.net
}

How to cite this paper: Bourdillon, A.J. (2018) Many-Body-Gravitational Solutions for Galactic Rotational Velocities, Including Possible Negative Mass. Journal of Modern Physics, 9, 1304-1316.

https://doi.org/10.4236/jmp.2018.96079

Received: April 27, 2018

Accepted: May 22, 2018

Published: May 25, 2018

Copyright $\odot 2018$ by author and Scientific Research Publishing Inc. This work is licensed under the Creative Commons Attribution International License (CC BY 4.0).

http://creativecommons.org/licenses/by/4.0/

\section{(c) (i) Open Access}

\begin{abstract}
The necessary derivation of negative mass in dispersion dynamics suggests cosmic applications. The method analyzes functional relationships between particle angular frequency, wave vector, rest mass and electromagnetic or nuclear potential, $f\left(\omega, k, m_{0}, V\right)=0$. A summary of consequential predictions of the dynamics leads to a calculation of ways in which negative mass might influence such phenomena as the rotational velocities that are observed in spiral galaxies. The velocities are found to be not Newtonian in the simple two body approximations for our solar system; but nearly constant with increasing orbital radii. It has moreover been suggested that the motion is due to halo structures of dark matter or dark energy. However, the motion is simply described by many-body gravitation that is transmitted along elastic spiral arms. In this context, we calculate possible effects of negative mass, but without observational confirmation.
\end{abstract}

\section{Keywords}

Dispersion Dynamics, Negative Mass, Velocity Plateau, Spiral Galaxy, Many-Body Gravitation

\section{Introduction}

Dispersion dynamics [1] [2] [3] [4] [5] is based on the formula in special relativity which contains the functional relationship between energy $E$, momentum $\boldsymbol{p}$ and rest mass $m_{o}$ of a free body, $f\left(E, p, m_{\mathrm{o}}\right)=0$. In wave mechanics, this translates to $f\left(\omega, k, m_{0}, V\right)=0$, by substitution with angular frequency in Planck's law; with wave vector in the de Broglie hypothesis; and with potential $V \neq 0$ for a bound particle. An immediate consequence is that the product of the group 
velocity and phase velocity in a free particle is equal to the square of the speed of light $c^{2}$. Further derivation will show (described below) that antiparticles have negative mass. This concept has a history of speculation [6] and it is not known for sure whether the gravitational force between matter and antimatter is attractive or repulsive. Lack of massive, observational, annihilation events suggests that antimatter is either a comparatively rare constituent in the universe, or that the particle-antiparticle gravitational force is repulsive, which would make massive particle annihilation improbable in cosmic terms. In this paper we consider what effect negative mass might have among the phenomena of rotational velocities in spiral galaxies [7].

Prior expectation supposed that central attraction in a galaxy would result in Newtonian planetary velocities where the inverse square gravitational attraction balances centrifugal forces due to orbital motions: then a planet of mass $m$ orbiting a sun $M$ at radius $\sim r$ reacts against the Gravitational attraction $G M m / r^{2}$, where $G$ is the gravitational constant. The planet in an approximately stable orbit would have a velocity $v=\sqrt{G M / r}$ i.e. inversely proportional to the square root of the orbital radius, for simplicity supposed circular. In fact, the velocities that are observed are almost independent of $r$ [7]. To some researchers, this implies existence of dark matter that increases the central attraction on visible matter in the galaxy.

\section{Dispersion Dynamics in Summary}

\subsection{Wave-Particle Duality}

The most fundamental property of modern physics is wave-particle duality. It is best expressed by the stable wave packet: self-evidently stable as the travelling wave group for a free particle or photon:

$$
\phi=A \cdot \exp \left(\frac{X^{2}}{2 \sigma^{2}}+X\right), \text { with } X=i(\bar{k} x-\bar{\omega} t)
$$

(Figure 1). The variables are stable not only because they are mean values of a symmetric wave function; but they are guaranteed stable by respective conservation of energy and momentum; and triple guaranteed by symmetry in space-time. In the direction of propagation, $X$ is an imaginary variable that causes $\phi$ to oscillate. The denominator $\sigma$ is particular because it depends on initial conditions but it is stable during propagation in free space as a consequence of Newton's first law of motion. The normalizing amplitude $A$ depends on the coherence $\sigma$ and is therefore equally stable. The envelope, $\exp \left(X^{2} / 2 \sigma^{2}\right)$, depends on the square of $X$ which is a function of four variables. Two are already considered, so we are left with the variables $x$ and $t$ that describe the profile. Since the other variables are all stable, this profile is also stable. From the start of quantum mechanics, and following Dirac's opinion [8], it has always been supposed that the wave packet is unstable; but now, with a stable wave group, we take a new perspective. 


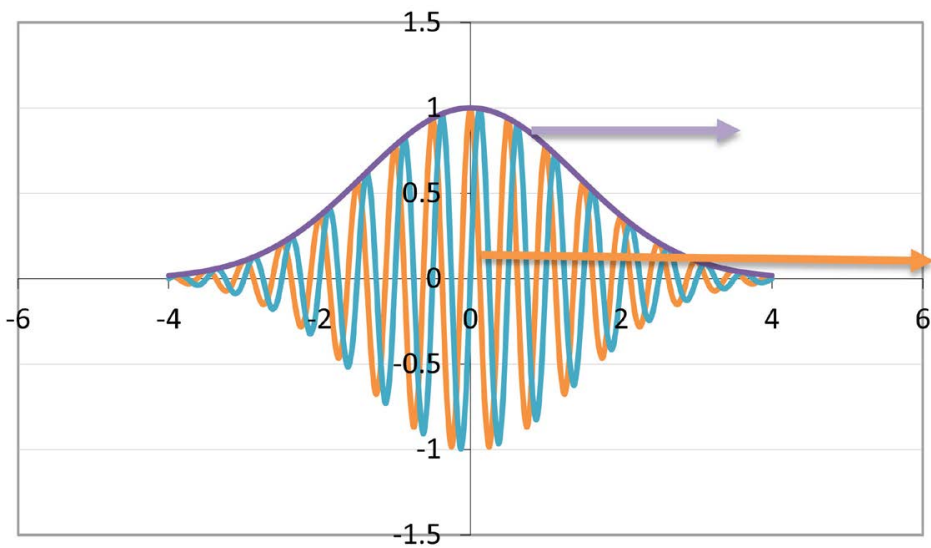

Figure 1. Stable wave packet (Equation (1)) containing envelope with group velocity $v_{g}$ (blue arrow) and real (orange) and imaginary (blue) parts of the carrier wave with phase velocity $v_{p}$ (orange arrow) .

\subsection{Solutions for the Particle Function $f\left(\omega, k, m_{0}, V\right)=0$}

From these wave mechanics are also derived Planck's law, $E=\hbar \omega$, the de Broglie hypothesis $p=\hbar k$, and several conservation rules. Solve $f\left(\omega, k, m_{0}, V\right)=0$, first for the free particle with rest mas $m_{o}$ in a potential $V=0$.

Operation of the relativistic Klein-Gordon equation, $\left(\square^{2}-m_{0}^{2}\right) \phi(x)=0$, on Equation (1) yields, as output, an algebraic equation in second order:

$$
\hbar^{2} \omega^{2}=\hbar^{2} k^{2} c^{2}+m_{0}^{2} c^{4}
$$

$\hbar$ being the reduced Planck constant and $c$ the speed of light. This is the same equation as is obtained from Einstein's relativistic formula, $E^{2}=p^{2} c^{2}+m_{0}^{2} c^{4}$, by substituting for energy using Planck's law and for momentum using the de Broglie hypothesis. The equation can be simplified with appropriate units $c=1$ $=\hbar$. Differentiation then gives a new result in relativity, for the product of group velocity $\mathrm{d} \omega / \mathrm{d} k$, (see e.g. [1]) and (more obviously) phase velocity $\omega / k$.

$$
\frac{\mathrm{d} \omega}{\mathrm{d} k} \cdot \frac{\omega}{k}=v_{g} \cdot v_{p}=1 \quad\left(=c^{2} \text { in generalized units }\right)
$$

The result is plotted in the positive quadrant of Figure 2 for the case rest mass $m_{0}=1$. The group velocity is well behaved: it tends to zero at low $k$ and to $c$ at large $k$ exactly as in the special theory of relativity ${ }^{1}$. The phase velocity is faster than the speed of light $c$ and is singular when $k \rightarrow 0:$ within this rest frame, time is Newtonian within the coherence $\sigma$. This has significance in the reduction of the wave packet during a quantum transition [1].

\subsection{Quantization}

The Uncertainty Principle can be derived from Equation (1) by Fourier transforms [1]. The derivation accounts for negative uncertainties, as occurs in Fresnel diffraction. Then Quantum physics is a consequence of wave motion, together with spatio-temporal constraints on bound states-most obviously the

${ }^{1}$ Those who calculate the speed of the electron should know which velocity they mean to calculate. 


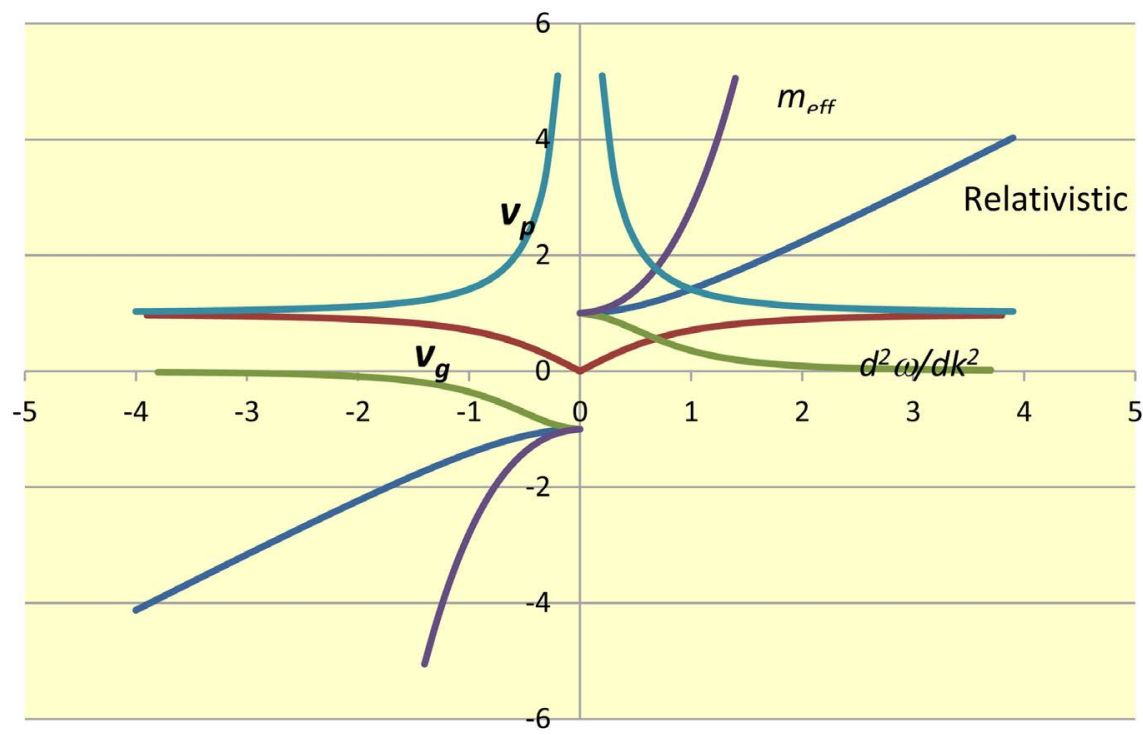

Figure 2. Functions for a free particle in Dispersion Dynamics, where $f\left(\omega, k, m_{0}, V\right)=0$, plotted against abscissae wave vector $k$. Ordinates are a mathematical set, being the ratios of the various physical quantities/unit values, including the energy of a relativistic free particle where $\hbar \omega=m^{\prime} c^{2}=\left(k^{2}+m_{0}^{2}\right)^{1 / 2}$ in case $m_{0}=1$ (using units $c=1=\hbar$ ); phase velocity $v_{p}=\omega / k$, group velocity $\mathrm{d} \omega / \mathrm{d} k$, dispersive curvature $\mathrm{d}^{2} \omega / \mathrm{d} k^{2}$; and effective mass $m_{\text {eff }}=\left(\mathrm{d} v_{g} / \mathrm{d} k\right)^{-1}$. Negative mass in antiparticles is the necessary solution for the unphysical singularity when, alternatively, $\hbar k=-m_{0} c$. The antiparticles are plotted with negative $k$, being an alternative representation for the Feynman-Stückelberg switching principle.

states involved in atomic spectral line emissions or absorption. Without quantization, as in the Bohr atom, wave functions would destructively self-interfere. Notice that in the calculation of lines such as the Lyman $\alpha$ for the hydrogen atom, $m_{o}$ cancels.

Two important facts are: the expected mass energy or density, integrated in time over the packet in Equation (1), is equal to $\hbar \bar{\omega}$; while the expected momentum, integrated over space, is equal to $\hbar \bar{k} \quad[1]$.

This description is a physical, non-axiomatic ${ }^{2}$ [9] definition of quantization.

\subsection{Velocity}

Dirac's calculation for the speed of the electron [8] found it equal to $c$. This contradicts relativity, as does his “jitter". By contrast, the phase velocity, $\omega / k$, brings clarity. It breaks the light barrier. Can it be measured? Yes, as the inverse of the group velocity, or as the ratio of energy to momentum. But is it real? No: for spatio-temporal reasons the energy in the packet is $\hbar \omega$, but the wave function is complex and energy is carried by the absolute group, $\int \phi^{*} \phi \mathrm{d} \tau$; not by the phase. What has to be understood is how peaks in the complex carrier wave-whether

${ }^{2}$ Mathematics creates parallel universes by the method of axiom and theorem; physics discovers the real world by the method of hypothesis and falsification. Axioms are logically true always; hypotheses are meaningless when unfalsifiable, and true only until falsified [9]. The mathematics must be consistent; hypotheses may be contradictory, though typically contradiction is reduced after falsification. 
real or imaginary-appear, grow, pass through the group, and disappear, as if elastic. Their importance lies in superposition and interference.

\subsection{Highly Relativistic and Non-Relativistic Approximations}

Two extreme regimes are commonly identified: relativistic when $m_{0} c \ll p$, and non-relativistic when $m_{0} c \gg p$.

Relativistically, at high $k \gg m_{0}$, both the group velocity and phase velocity tend to the speed of light: $V_{g}, V_{p} \rightarrow c$, as in the massless photon travelling in free space. Then $\mathrm{d} \omega / \mathrm{d} k=\omega / k=v^{\prime} \lambda$, the product of oscillational frequency $v^{\prime}$ with wavelength. Conductance depends on the group velocity.

At low $k \ll m_{0}$, non-relativistic values approximate:

$$
E=m_{0}\left(1+p^{2} c^{2} /\left(m_{0}^{2}\right)\right)^{1 / 2} \approx m_{0}+p^{2} / 2 m_{0}
$$

In classical mechanics, the mass energy is ignored as a constant in mechanical or chemical changes, as it is in Schrödinger's equation-which is likewise non-relativistic. Moving on from the free particle, when the potential $V \neq 0$ is included, the Schrödinger eigenvalue $\varepsilon$ corresponds to the result of the virial theorem, so that the expectation value for $\langle|V|\rangle \approx-2\left\langle\left|p^{2} / 2 m_{0}\right|\right\rangle$ (Figure 3) and:

$$
\varepsilon \approx\left\langle\left|\hbar^{2} k^{2} / 2 m_{0}\right|\right\rangle
$$

Using the simplified units previously described. The kinetic energy for the free particle is positive; while the eigenvalue in a potential $V \neq 0$ is about the same absolute value, but negative. Whether free or bound, the group velocity (and conductance etc.) is given by:

$$
C=\frac{E-m_{0}}{2 p}=\frac{\omega-m_{0}}{2 k}
$$

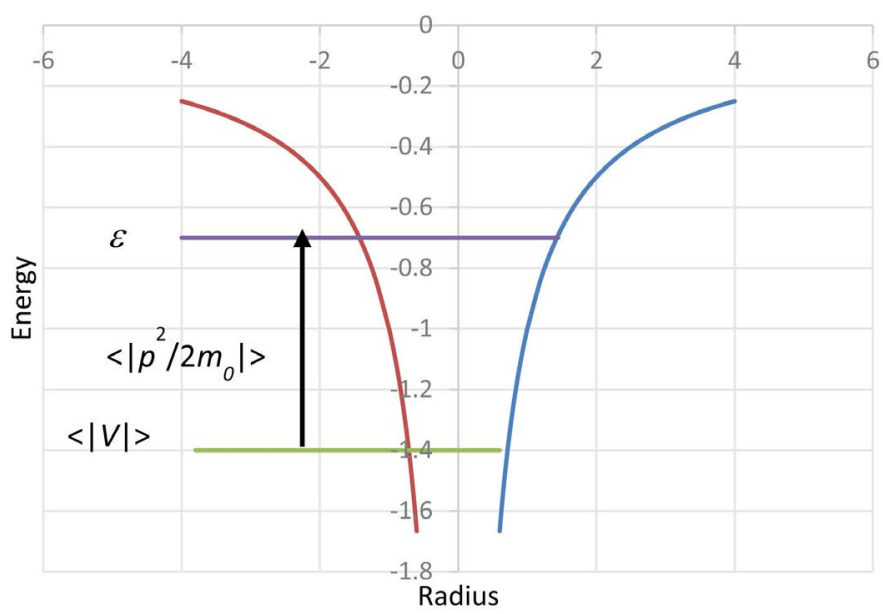

Figure 3. Following the Virial theorem, the expectation value for the potential energy is double the expectation for the kinetic energy on, for example, an electron bound by a Coulomb potential. The difference is the eigenvalue $\varepsilon$. In Dispersion Dynamics, the non-relativistic group velocity is approximately half the ratio of the energy/momentum, $v_{g} \rightarrow \mathcal{E} / 2 p$, as in classical mechanics. However, when $k \gg m_{0}$, then $v_{g} \rightarrow k / \omega \approx c$. 
or $\varepsilon / 2 p$ in Schrödinger's system. This is the velocity that is proportional to the Lorentz force of magnetism for a charged particle moving in free space.

In constrained crystal fields, the force depends on the dispersive curvature $\mathrm{d}^{2} \omega / \mathrm{d} k^{2}$ of electron energy bands near the Fermi surface [1]. The second derivative therefore controls the sign of the Hall coefficient $R_{H}$. For this reason, $R_{H}$ is positive in $\mathrm{Al}$, though in the majority of metals it is negative, as in $\mathrm{Cu}$.

\subsection{Why Is Negative Mass Necessary?}

The analysis of the function $f\left(\omega, k, m_{0}, V\right)=0$ summarized above, modifies the conventional notion of rest mass $m_{0}$. Equation (2) has solutions when energy is positive or negative. In the Schrödinger equation, the energy is negative since the expectation value for the potential $\langle|-V|\rangle$ is negative, but, in value, double the expectation for kinetic energy $\left\langle\left|p^{2} / 2 m_{0}\right|\right\rangle$, which is positive. This is typically the case for bound states in atoms. However, for the free particle, when $V=0$, there remain positive and negative solutions. In his relativistic equations, Dirac ascribed his negative eigenvalues to antiparticles, and we follow him in so ascribing the negative solution for energy in Equation (2). Two consequences follow: firstly, when $k=0$, in the rest frame, the result for relativistic mass $E=m^{\prime} c^{2}$ implies that for the antiparticle, both $m$ ' and $m_{0}$ are negative. Secondly the supposed alternative that $E<0$ while $m>0$ leads to anomalies when $|k|=\left|m_{0}\right|[1]$, Appendix A.II]: the condition would imply a singularity in $v_{g}$ combined with zero net energy and zero $v_{p}$. Neither of these consequences is observed, and the condition is generally inconsistent with physical theory. Fundamental conclusions of Dispersion Dynamics are that the antiparticle has negative mass, and also that they have negative kinetic energy. This solution for the equation is a simple convention that supplies consistency and is clearer than Dirac's concept of hole states which is not generally favored [10]. The solution is like conventional metallic conduction by negatively charged counter currents: it does not change the way we work with positrons in particle accelerators.

However, the consistency reopens some old doubts. With a new representation for the wave function of antiparticles, we also find a non-paradoxical explanation for the Feynman-Stückelburg principle: "An antiparticle travelling forward in time does not exist". Though stated to explain transition and scattering probabilities, the principle seems to contradict cloud chamber and bubble chamber images of particle creation. By contrast, Dispersion Dynamics has, "An antiparticle travelling forward in time has negative momentum." This causes the same arrow reversal in Feynman diagrams as before. However, it implies also consistency with the physically constant electronic charge to mass, ratio $\mathrm{e} / \mathrm{m}$, i.e. having the same sign for both charged particle and antiparticle.

\section{Galactic Rotation Velocities Due to Many-Body Gravitation}

\subsection{Newton's Law of Gravitation between Two Bodies}

The best known part of the universe is our own solar system. Newton gave us the 
law of gravitation for planetry motion, to which perturbations due to general relativity are added. Galactic orbits are more complicated. Their rotational velocities do not follow, in simple form, Newton's law of gravitation [7]. Typically, except close to the galactic center, orbital velocities are comparatively independent of galactic radius.

We get an idea of the force by comparing with electrostatics, which also follows the inverse square rule, i.e. proportional to $r^{-2}$. Both forces operate between two bodies; but in electrostatics we are used to applying many-body interactions. Planetry forces are comparatively simple: the sun on the planets, the moon on the earth etc. The orbit of the moon around the earth is perturbed by the distant sun; producing a wobble on the earth's orbit. In galaxies by contrast, matter that orbits a galactic center is influenced also by other masses, some nearer; others further away. The observational fact that they mostly travel with similar velocities, implies multiple forces that are more than perturbations. Consider the gravitational forces between three masses: a solar center $M$, and two orbiting masses, $m_{1}$ and $m_{2}$, traveling at similar velocities on the same plane. At a moment when the three masses lie sequentially on a straight radial line, the centripetal forces are $G M m_{1} / r_{1}^{2} ; G M m_{2} / r_{2}^{2} ; G M m_{3} / r_{3}^{2}$; and $-G M m_{1} m_{2} /\left(r_{1}-r_{2}\right)^{2}$. The last has a centrifugal reaction on $m_{2}$ (since $r_{3}>r_{2}$ ) that adds to inertia opposing the central force. Where there are a large number $n$ of masses, moving with similar velocities far from the galactic center, i.e. with $m_{1} /\left(r_{1}-r_{n}\right)^{2}>M / r_{1}^{2}$ etc., a many-body resultant force might be written, similar to electrostatics:

$$
F=G M m_{1} / e_{g} r^{2}
$$

where $e_{g}$ is a gravitational permittivity. This would not be a material property but a consequence of environment. We could use gravitational permittivity in the context of gravitational lensing; but meanwhile we shall see how this many-body effect is due to galactic radius while causing and stabilizing it.

\subsection{Rotational Velocities}

Before proceeding to investigate what effect negative mass in antimatter might have on rotational velocities, we need to understand familiar positive mass in regular matter. We will then carry forward what we know about dispersion dynamics to speculation about gravity in antimatter.

\subsection{Method}

- For exploratory purposes, we suppose that dark matter is simply normal matter at typical temperatures with low visibility whether due to halo or other configurations, though possibly including by extension, uncharged particles, whether light or heavy.

- Start with a massive spherical core.

- Continue with a planar disc to represent smoothly distributed matter in a bright galaxy. 
- Let many-body gravitational bonding cause constant rotational velocities.

- Calculate the structure of the galaxy for general stability by considering the gravitational forces balanced against inertia under observed rotational constraints.

- Adapt the calculations for spiral arms supposing these are gravitationally coupled to adjacent matter

- Consider negative mass.

\subsection{Gravitational Attraction and Centrifugal Force}

Various measurements demonstrate approximately constant galactic rotational velocities [7], or velocities with linear dependence on radii. Our own solar sun, in the 4.5 billion years since its formation, has orbited its galaxy about 20 times. In consequence galactic spiral arms have wound onto a planar disc. On the Newtonian model, the balance of centrifugal inertia with net centripetal gravitation, implies the relationship $v \approx \sqrt{F / r}$ where the gravitational force:

$$
F=\Sigma G M(r) \stackrel{\text { supposedly }}{\approx} G \rho(r)
$$

is extended in the many-body case to a force that is, in a simple approximation, proportional to a generalized galactic density $\rho(r)$ that is treated as a continuum, and presumed to follow some power of radius, $r^{2}$. We need to calculate what value of a will provide a constant galactic rotational velocity. The rotational velocities will then depend accordingly, $v(r) \approx G \rho(r) / r$. Begin by examining the forces acting on an elemental ring in the galactic disc.

\subsection{Many-Body Planar Gravitation}

We treat the galaxy as approximately planar to which is added a spherical hub (Figure 4). This is important in the context, because the intuitions provided by symmetric systems under Gauss's theorem are here misleading. Assume, for simplicity, cylindrical symmetry in the disc. Then divide the galaxy into rings of increasing radius. Analyze one ring at the start, and then sum over all rings in the galaxy. To substitute reasonable cut-offs for singularities at small $r$, use finite

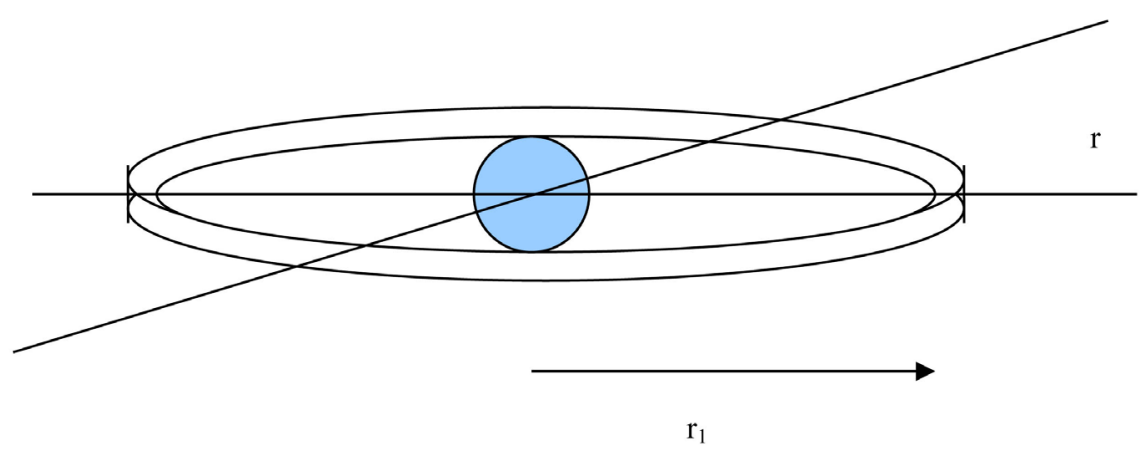

Figure 4. Illustration showing an elemental ring, radius $r_{1}$, of matter inside a planar galaxy with spherical hub (filled circle) at center. The radius corresponds to 10 abscissae units in Figure 5. 
differentials instead of integrated infinitesimals. Then, along a given axis, the gravitational force $F(r)$ due to a ring of mean radius $r_{1}$, can be calculated from the formula:

$$
F(r)=2 G \rho\left(r_{1}\right) \sum_{\theta=0}^{\pi} \frac{\cos \left(a \tan \left(r_{1} \sin (\theta) /\left(r-r_{1} \cos (\theta)\right)\right)\right)}{\left(r-r_{1} \cos (\theta)\right)^{2}+r_{1}^{2} \sin ^{2}(\theta)} \Delta \theta \cdot \Delta r
$$

where $\rho(r)$ is the mass density in the ring. As is well known in electrostatics, the potential inside a conducting sphere is zero; the elemental ring is different. At first view, the result shown in Figure 4 does not support the common assumption that a halo of dark matter causes rotational velocities in galaxies to plateau. In particular, if the dark halo is constituted from light particles at large radius it would have the opposite effect from that required to simulate observations; while if the halo is constituted from heavy particles at shorter radius, it would most likely produce peculiar radial structure in the observed velocities. In either case a law that dictates the distribution of the matter is needed and a first approximate rule is developed here.

Figure 5 illustrates two particular features of the gravitational force due to the ring. On the one hand, the force is compressive for surrounding matter; while by reaction, the ring tends to break apart due to both the tidal forces of the galactic center and to the attraction of outlying matter which bonds to the ring. The latter is facilitated by similar rotational velocities in surrounding matter. The calculation allows a criterion for stability in the galaxy as a balance of three types of force: principally the galactic central attraction; with the inertia that accompanies rotational velocities; and with the tension due to the gravitational bonds with neighboring matter. A condition for stability in the ring (Figure 4) is the distribution of material density in the galaxy: this minimizes the tension so that the sum of the depicted forces $F$, multiplied by the local mass density is zero,

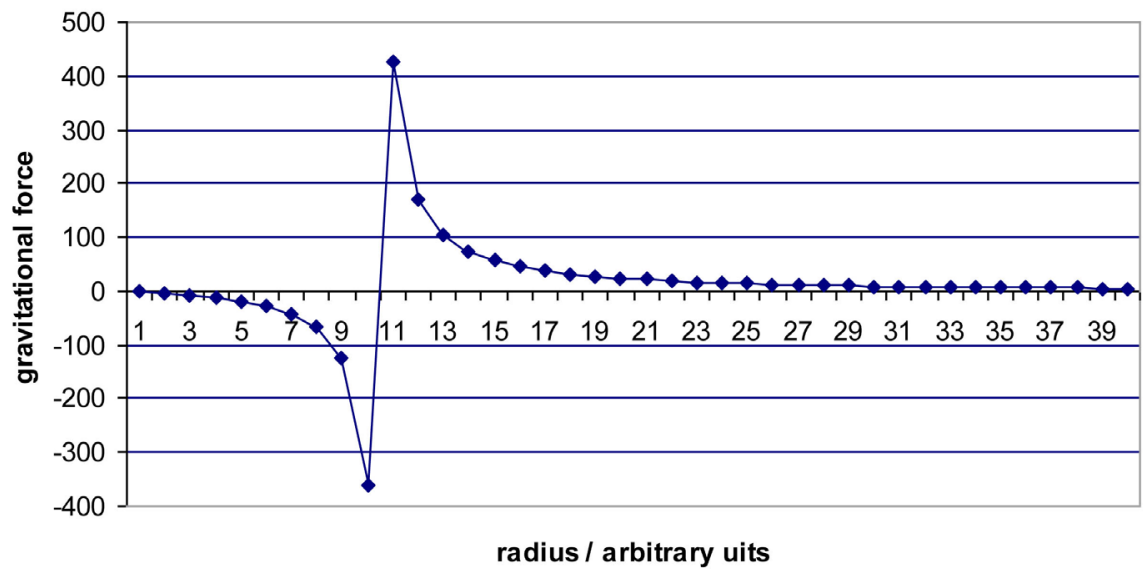

Figure 5. Gravitational force at radius $r$, due to an elemental ring of matter with mean radius $r_{1}=10$ units (Figure 4 ). The measurements are from the ring center outwards: negative values are centrifugal; positive values centripetal. A halo will produce a smoothed version of this configuration. The graph illustrates localization between elements of the many-body attraction. 
$\Sigma F(r) \cdot \rho(r) \rightarrow 0$. The density $\rho(r)$ can therefore be simulated by varying the index on the power law proportional to $r^{-1.15}$. Notice that this rule applies only to the neighboring matter; not to the central hub. When we add the central hub, the power law will change. Supposing stability, we can now return to calculate rotational velocities. The calculated value of $r^{2}$ gave a first estimate of the mean mass density distribution in the plane beyond the central hub. Adjustments must now be made to correct for two suppositions: the galaxies that are still evolving are not in equilibrium; and the central hub cannot be neglected, so is included in the following calculation.

The elemental rings illustrated in Figure 4 and Figure 5 produce gravitational forces whose net effects are calculated in Figure 6(a) and Figure 6(b). At short

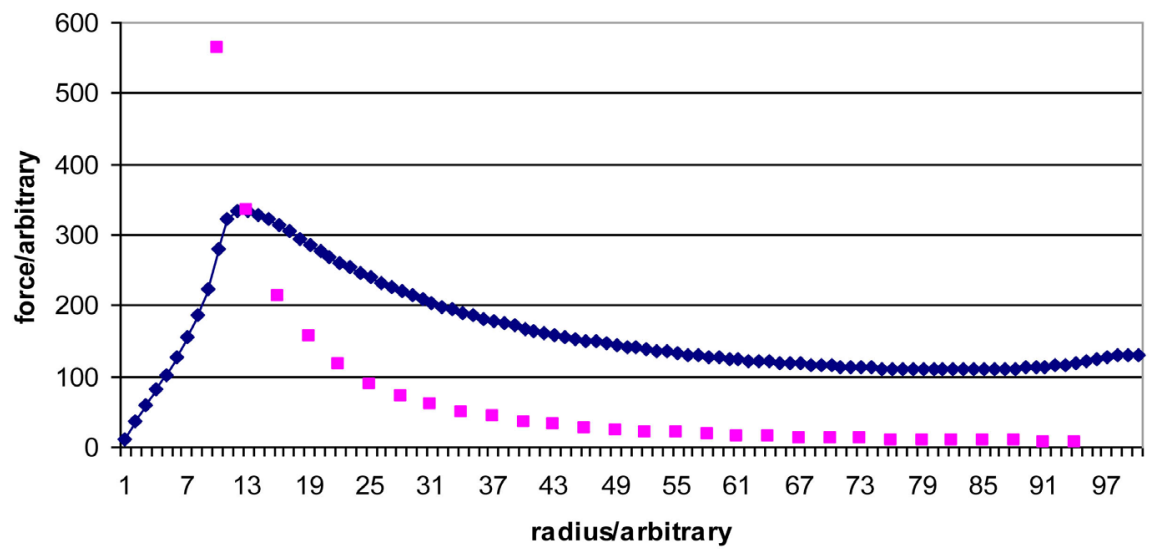

(a)

$$
\rho(r) \alpha r / r_{1} ; \rho(r) \alpha r^{1.1} / r_{1} ; \rho(r) \alpha r^{1.2} / r_{1}
$$

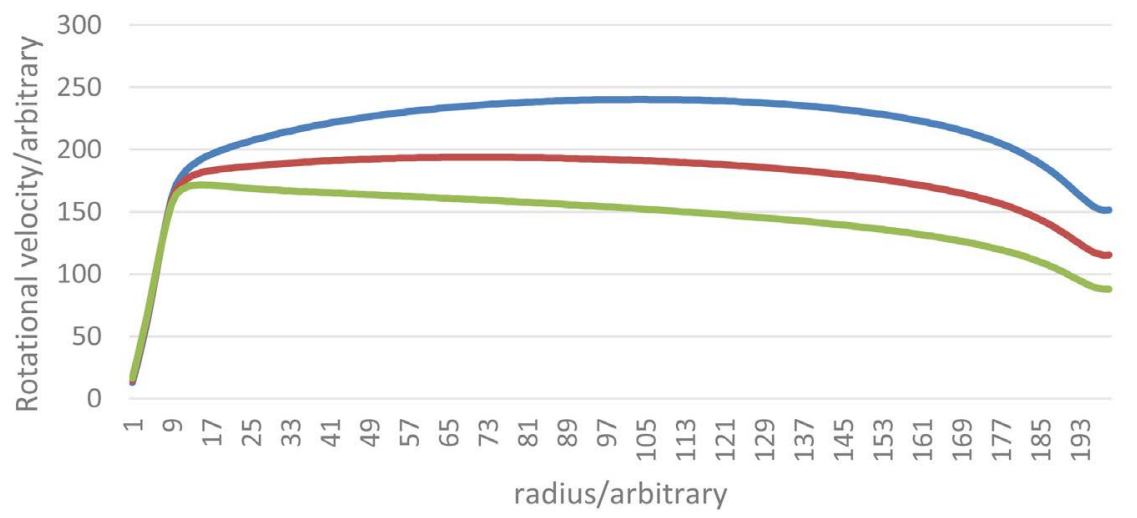

(b)

Figure 6. (a) Net many-body, gravitational, centripetal, force calculated from summed rings (line) on a plane with mass density distributed as $\rho(r)=1 / r$, the inverse of the radius. The force is multiplied by $r$ near the center to account for spherical galactic center. The terminal increase at $r>90$ is fallaciously due to the following truncation at $r=100$. The line is compared with the Newtonian inverse square law from a point source (isolated squares); (b) Calculated plateaux in rotational velocities $(F / r)$ due to many-body gravitation, calculated from summed elemental forces for planar mass density distribution increasing from uniform (green); to increasing indices $\sim P^{0.1}$ (red) and $\sim I^{0.2}$ (blue). 
radius, where the galactic center approximates, by supposition, to spherical symmetry, the zero potential is applied in Figure 6(a) and Figure 6(b) at their origins. Further out, the forces due to a set of such rings are summed. The terminal increase at large $r$ is a computational artifact due to truncation of the summation. It is ignored in the present study and will be taken up again later. The slope of the plateau is sensitive to detailed distribution of the mass density, especially to the power in $r^{2}$. Notice that the elemental volume, $\Delta r \cdot \Delta z \cdot r \Delta \vartheta$, depends on the first power of $r$, so that the summation represented in Equation (8) implies the mass density distribution $\rho \approx 1 / r$ if the elemental volume is treated as constant; or alternatively, the force converts to rotational velocity when $\rho$ is substituted realistically, i.e. when the variable function is formed to be constant as in Figure 6(b).

To simulate the gravitation on a spiral arm, the density is converted to a net resultant force on a particular body due to matter distributed about radii that are greater or less than its own.

\subsection{Negative Mass}

With the results so far given, next consider effects on rotational velocities due to spatial variations in matter and antimatter. Electromagnetic forces in charged particles are greater than gravitational forces, which typically therefore, are more difficult to measure. In uncharged systems, or in galaxies where negative and positive charges are neutralized, we suppose as before, that particles and antiparticles are gravitationally repulsive due to their opposite masses. Then the two types of matter are held apart by gravitational forces and massively explosive annihilations between colliding galactic structures are avoided. Meanwhile, contrary to the standard view that antimatter is rare and asymmetrical, suppose it is thinly distributed in space, but separated from matter by gravitational repulsion. To illustrate, Figure 7 indicates how antimatter might affect observable rotational velocities for the unlikely case that the antimatter extends the truncated plane that is used in Figure 7(a). The truncation occurs at channel 100, and velocities beyond this point are due to the gravitational attraction by the galactic plane and center. The arms of the galaxy are like elastic octopus tentacles that wrap and spin, while accreting material away from nearby passing structures.

By comparison, Figure 7(b) shows how the rotational velocities would be affected by continuation of the plane with antimatter. Beyond channel 100, the velocities pass through zero and reverse. Because this configuration is unlikely, we use these graphs to speculate on more likely scenarios. Assuming matter and antimatter are separated by gravitational repulsion, then a surrounding sea of antimatter will compress the galaxy in both its axial and radial directions. However if the sea is spherically symmetric, then Gauss' law shows that the gravitational potential that is due to it is zero. Antimatter in a sea of matter, will behave the same as matter within a sea of antimatter. 


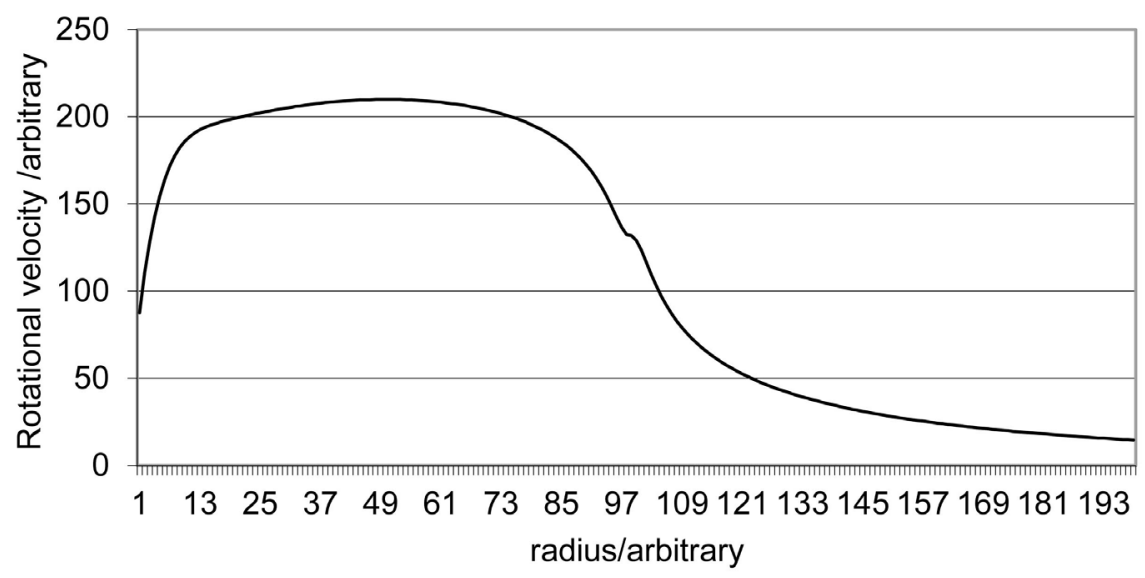

(a)

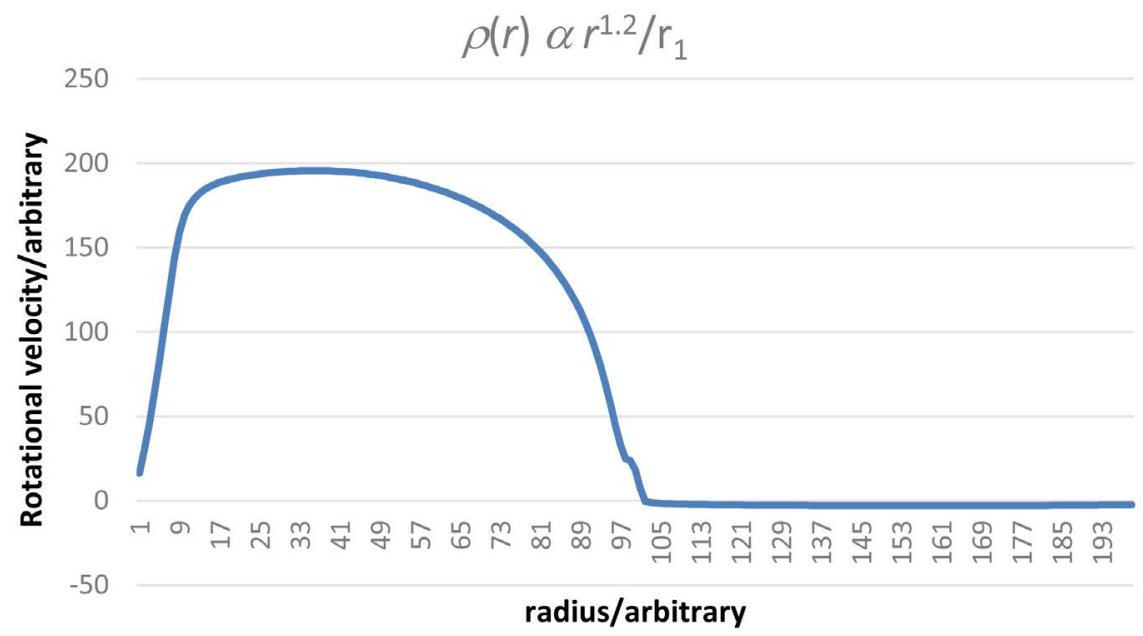

(b)

Figure 7. (a) Calculated rotational velocities when the planar density is $r^{1.2} / r_{1}$ and matter truncates at channel 100; (b) Calculated rotational velocity as a function of radius but with matter replaced with antimatter (supposedly repulsive) beyond $\mathrm{r}>100$ arbitrary units.

\section{Conclusion}

Many-body gravitation provides a simple explanation for observed plateaux in velocity/radius ratios in spiral galaxies. In contrast wave-particle duality, expressed in the stable wave packet, demonstrates the necessity for negative mass in particle physics. Invisible halos and other structures that might surround or imbue spiral galaxies, when analyzed by suppositions dependent on negative mass, are not conducive to the plateaux that have previously been regarded as anomalous. Many-body gravitation is a simpler explanation, for the plateaux that are observed in rotational velocities, than dark matter or dark energy [7]. The calculations imply that the swirling motion causes a small increase in matter density away from the central pull towards a galaxy axis.

\section{References}

[1] Bourdillon, A.J. (2017) Dispersion Dynamics in the Hall Effect and Pair Bonding in 
$\mathrm{HiT}_{c}$. Nova Science, New York.

[2] Bourdillon, A.J. (2012) Journal of Modern Physics, 3, 290-296. https://doi.org/10.4236/jmp.2012.33041

[3] Bourdillon, A.J. (2013) Journal of Modern Physics, 4 705-711. https://doi.org/10.4236/jmp.2013.46097

[4] Bourdillon, A.J. (2014) Journal of Modern Physics, 5, 23-28. https://doi.org/10.4236/jmp.2014.51003

[5] Bourdillon, A.J. (2017) Journal of Modern Physics, 8, 483-499. https://doi.org/10.4236/jmp.2017.84031

[6] Villata, M. (2011) Europhysics Letters, 94, 2. https://doi.org/10.1209/0295-5075/194/20001

[7] Rubin, V.C. (2006) Physics Today, 59, 8-9. https://doi.org/10.1063/1.2435662

[8] Dirac, P.A.M. (1958) The Principles of Quantum Mechanics. 4th Edition, Oxford.

[9] Popper, K.R. (1959) The Logic of Scientific Discovery. Hutchinson

[10] Charlton, M. and Humbertson, J.W. (2001) Positron Physics. CUP, Cambridge. 